\title{
RESPOSTAS NUTRICIONAIS DE CAFEEIROS CATUAÍ E ICATU A DOSES DE CALCÁRIO EM SUBSUPERFÍCIE ${ }^{(1)}$
}

\author{
Luciana Aparecida Rodrigues ${ }^{(2)}$, Hermínia Emilia Prieto Martinez ${ }^{(3)}$, Júlio César \\ Lima Neves ${ }^{(4)}$, Roberto Ferreira Novais ${ }^{(4)}$ \& Sebastião Marcos de Mendonça ${ }^{(5)}$
}

\begin{abstract}
RESUMO
O aprofundamento do sistema radicular no solo é condicionado por relações $\mathrm{Ca} / \mathrm{Al}$ adequadas, podendo a calagem subsuperficial apresentar efeito positivo no crescimento das raízes e na absorção de nutrientes. Assim sendo, foi realizado um experimento em colunas de solo, em casa de vegetação, no qual se avaliou a absorção de Al, Ca, Mg e P por duas variedades de cafeeiros (Catuaí e Icatu), uma sensível e outra tolerante ao $\mathrm{Al}$, respectivamente, em função da aplicação em subsuperfície de sete doses de calcário $\left(0,0 ; 0,49 ; 1,7 ; 2,9 ; 4,1 ; 6,6\right.$ e $\left.9,3 \mathrm{t} \mathrm{ha}^{-1}\right)$. Cultivaram-se as plantas até 6,5 meses de idade, em solo acondicionado em colunas de PVC, subdivididas em três anéis. $O$ anel superior recebeu calcário e fertilização. Nos dois anéis inferiores, as saturações por $\mathrm{Al}$ (m \%) variaram de 0 a 93 \%. A aplicação de calcário na subsuperfície aumentou os teores de Ca e Mg na parte aérea e nas raízes e o teor de $P$ nas folhas superiores de ambas as variedades. A eficiência de utilização de Ca na parte aérea e em raízes decresceu com a aplicação do calcário em subsuperfície para ambas as variedades, enquanto a eficiência de utilização de $P$ diminuiu somente para a parte aérea da var. Icatu. A aplicação do calcário na subsuperfície reduziu o teor de Al na parte aérea da var. Icatu e em raízes da var. Catuaí. Os teores de $\mathrm{P}$, Ca e Mg nas folhas foram adequados às variedades, independentemente da quantidade de calcário aplicada ao solo, indicando que a adubação e a correção da acidez da camada superficial do solo foram eficientes para manter a planta nutrida, independentemente do teor de Al na subsuperfície do solo.
\end{abstract}

Termos de indexação: calagem subsuperficial, nutrição mineral do cafeeiro, tolerância ao Al, toxidez por Al.

\footnotetext{
(1) Parte da Tese de Mestrado do primeiro autor, apresentada a Universidade Federal de Viçosa - UFV. Recebido para publicação em setembro de 2004 e aprovado em novembro de 2006.

(2) Professora da Fundação de Apoio à Escola Técnica - FAETEC. Instituto Superior de Tecnologia em Ciências Agrárias. Av. Wilson Batista s/n, CEP 28070-620 Parque Aldeia, Campos dos Goytacazes (RJ). E-mail: lua@uenf.br

(3) Professora do Departamento de Fitotecnia da Universidade Federal de Viçosa - UFV. Av Peter Henry Rolfs, s/n, CEP 36570000 Viçosa (MG). E-mail: herminia@ufv.br

(4) Professor do Departamento de Solos, UFV. E-mail: rfnovais@ufv.br; julio_n2003@yahoo.com.br

(5) Gerende de Pesquisa da Heringer. Rod. BR 262 Km 39, CEP 36900-000 Manhuaçu (MG). E-mail: sebastiao.mendonca@heringer.br
} 


\title{
SUMMARY: NUTRITIONAL RESPONSE OF CATUAÍ AND ICATÚ COFFEE PLANTS TO SOIL SUBSURFACE LIMING
}

\begin{abstract}
The Ca/Al ratio is determinant for root deepening in the soil subsurface layer. Liming this layer can improve root growth and nutrient absorption. Thus, a greenhouse trial was carried out using soil columns. Two varieties of coffee plants (Catuaí and Icatú), one Alsensitive and the other Al-tolerant, respectively, were evaluated in limed and surfacefertilized soil, whereas $0.0 ; 0.49 ; 1.7 ; 2.9 ; 4.1 ; 6.6$ and $9.3 t$ ha $^{-1}$ of limestone were applied to the subsurface soil. Plants were cultivated for 6.5 months in soil accommodated in PVC columns, subdivided in three rings. In the two lower rings $A l$ saturation varied from 0 to $93 \%$. Limestone application in the subsurface raised the Ca and $\mathrm{Mg}$ contents in the shoot and roots, and $P$ concentration in the upper leaves of both varieties. Following subsurface limestone application, Ca utilization efficiency decreased for both varieties, by the shoot as well as by roots. The $P$ utilization efficiency by the shoot decreased only in the Icatú variety. Subsurface limestone application reduced the Al content in the aerial part of Icatú and in Catuaí roots. The leaf concentration of $\mathrm{P}, \mathrm{Ca}$ and $\mathrm{Mg}$ remained at adequate levels in both varieties, indicating that fertilization and acidity correction of the surface soil layers were efficient at maintaining the plant nutrient levels, independent of Al levels in the soil subsurface.
\end{abstract}

Index terms: subsurface liming, coffee tree nutrition, Al tolerance, Al toxicity.

\section{INTRODUÇÃO}

Ainda que Coffea arabica não seja considerada uma espécie vegetal muito sensível ao $\mathrm{Al}$, Rodrigues et al. (2001) demonstraram que altas saturações do elemento restringem o desenvolvimento de raízes nas camadas subsuperficiais do solo, mesmo que a superfície esteja devidamente corrigida e adubada. Têm-se verificado graus distintos de tolerância ao $\mathrm{Al}$ para várias espécies agrícolas, até entre genótipos dentro de uma mesma espécie (Silva, 2002). Já foram observadas variações nos padrões de absorção de nutrientes e no crescimento entre genótipos de cafeeiros cultivados em condições de altas saturações por Al (Braccini, 1995, Mauri et al., 2004; Mattilello et al., 2004). Braccini et al. (1998a), avaliando genótipos de cafeeiros cultivados em solução nutritiva com $\mathrm{Al}$, observaram que a redução da produção da matéria seca das raízes foi a característica que permitiu melhor discriminação entre os genótipos.

Os efeitos deletérios do $\mathrm{Al}$ na modificação dos padrões de absorção e de metabolismo de nutrientes dependem da espécie vegetal, cultivar e das condições edáficas. A disfunção da membrana plasmática, ocasionada por alterações em sua estrutura, tem sido a causa principal dos padrões diferenciados de acúmulo de minerais em plantas sob estresse causado pelo $\mathrm{Al}$ (Furlani, 1989). De acordo com Huang et al. (1992), o $\mathrm{Al}$ inibe o influxo do $\mathrm{Ca}^{2+}$ através da plasmalema radicular, em virtude de um bloqueio nos canais de $\mathrm{Ca}$, ou alterando o transporte desse nutriente nas raízes e seus processos homeostáticos. Segundo Rengel et al. (1995), o bloqueio diferencial nos canais de Ca pode ser a chave da tolerância diferencial ao $\mathrm{Al}$.
Pavan \& Bingham (1982) observaram que a toxidez de $\mathrm{Al}$ em cafeeiro cultivado em solução nutritiva foi caracterizada pela redução na absorção não somente de $\mathrm{Ca}$, mas também de $\mathrm{Mg}$ e $\mathrm{P}$, e pelo aumento na absorção de $\mathrm{K}$ e $\mathrm{Al}$. No caso do $\mathrm{P}$, o $\mathrm{Al}$ solúvel reage com este elemento formando fosfato de $\mathrm{Al}$, que apresenta baixa solubilidade. Na planta, o Al pode imobilizar o $\mathrm{P}$ por reação de adsorção ou precipitação, fixando-o nas superfícies celulares e espaço livre aparente ou internamente na célula (Clarkson, 1966). Braccini et al. (1998b) observaram que a tolerância ao $\mathrm{Al}$ em cafeeiro foi associada ao menor acúmulo de $\mathrm{P}$ nas raízes.

Um fator importante a ser considerado é que, no campo, enquanto a superfície do solo é adubada e corrigida quanto à acidez, a subsuperfície permanece nas condições naturais do solo, apresentando, muitas vezes, alta saturação por $\mathrm{Al}$ e baixa saturação por bases (Guimarães \& Lopes, 1986). Rodrigues et al. (2001), avaliaram as respostas radiculares de mudas de cafeeiros em solo com a camada superficial corrigida quanto à acidez e submetido à calagem diferencial na profundidade de $12-34 \mathrm{~cm}$. Os autores verificaram redução no comprimento e na superfície das raízes na subsuperficie do solo em condição de alta saturação por Al. Esta resposta foi mais acentuada na variedade Catuaí, indicando a maior sensibilidade desta variedade ao $\mathrm{Al}$, em relação à variedade Icatu, o que foi verificado também por Braccini et al. (1996).

Uma vez sendo verificada que a presença do $\mathrm{Al}$ na subsuperfície leva variedades de cafeeiros sensíveis ao $\mathrm{Al}$ a apresentar raízes mais curtas e mais grossas (Rodrigues et al., 2001), sintomas típicos de toxidez de Al (Foy, 1974; Pavan \& Binghan, 1982; Furlani, 
1989; Braccini, 2000; Silva et al., 2002), é importante avaliar o quanto estas respostas influem na nutrição mineral das plantas, tendo em vista que este fator pode afetar a produção do cafeeiro.

O presente trabalho teve como objetivo avaliar a absorção de Al, P, Ca e Mg e a eficiência de utilização de $\mathrm{Ca}$ e $\mathrm{P}$ por genótipos de cafeeiros sensíveis e tolerantes ao $\mathrm{Al}$, em solo com calagem e fertilização na superfície e na presença de níveis de saturação por $\mathrm{Al}$ na subsuperfície.

\section{MATERIAL E MÉTODOS}

O experimento, realizado em casa de vegetação do Departamento de Fitotecnia da UFV, Viçosa, MG, constou de um delineamento em blocos ao acaso, em esquema fatorial 7 x 2 (sete doses de calcário na subsuperfície e duas variedades de café), com quatro repetições.

Cultivaram-se duas variedades de cafeeiro, uma sensível (Catuaí) e outra tolerante (Icatu) a Al (Braccini et al., 1996), em colunas de PVC de $20 \mathrm{~cm}$ de diâmetro, subdivididas em três anéis, correspondendo a três camadas de solo, de 0-12, 12-24 e 24-34 cm de profundidade. Utilizou-se um Latossolo Vermelho Amarelo da região de Viçosa, MG com classificação textural muito argilosa (29\% areia, $3 \%$ silte e $68 \%$ argila) e densidade de $1,1 \mathrm{~kg} \mathrm{dm}^{-3}$. O solo empregado apresentava, originalmente, as seguintes características químicas: pH (água, 1:2,5) 3,9; m = $93 \% ; \mathrm{C}=3,5$ dag kg $^{-1} ; \mathrm{P}=2,8 \mathrm{mg} \mathrm{dm}^{-3}$ (Extrator Mehlich-1); $\mathrm{K}^{+}=8,0 \mathrm{mg} \mathrm{dm}^{-3}$ (Extrator Mehlich-1); $\mathrm{Ca}^{2+}=2,2 \mathrm{cmol}_{\mathrm{c}} \mathrm{dm}^{-3}$ (Extrator $\mathrm{KCl} 1 \mathrm{~mol} \mathrm{~L}^{-1}$ ); $\mathrm{Mg}^{2+}$ $=0,44 \mathrm{cmol}_{\mathrm{c}} \mathrm{dm}^{-3}\left(\right.$ Extrator KCl $\left.1 \mathrm{~mol} \mathrm{~L}^{-1}\right) ; \mathrm{H}+\mathrm{Al}=$
$7,5 \mathrm{cmol}_{\mathrm{c}} \mathrm{dm}^{-3}$ (Extrator $\mathrm{Ca}(\mathrm{OAc})_{2} 0,5 \mathrm{~mol} \mathrm{~L}^{-1} \mathrm{pH} 7,0$.); $\mathrm{V}=4 \% ; \mathrm{Al}$ trocável $=1,4 \mathrm{cmol}_{\mathrm{c}} \mathrm{dm}^{-3}$ (método do aluminon).

$\mathrm{O}$ solo referente à camada superior $\left(3,77 \mathrm{dm}^{3}\right) \mathrm{de}$ todos os vasos recebeu 8,8 $\mathrm{t} \mathrm{ha}^{-1} \mathrm{de} \mathrm{CaCO}_{3}+\mathrm{MgCO}_{3}$ (relação estequiométrica 4:1) correspondendo a $1,9 \mathrm{vez}$ a necessidade de calcário. O solo dos dois anéis inferiores $\left(6,91 \mathrm{dm}^{3}\right)$ recebeu sete doses $\mathrm{CaCO}_{3}+$ $\mathrm{MgCO}_{3}$ (relação estequiométrica 4:1, $131 \% \mathrm{PRNT}$ ): 0,$0 ; 0,49 ; 1,71 ; 2,92 ; 4,15 ; 6,58$ e $9,30 \mathrm{t} \mathrm{ha}^{-1}$, correspondentes a 0,$0 ; 0,1 ; 0,37 ; 0,64 ; 0,9 ; 1,45$ e 2,0 vezes a necessidade de calagem, para elevar a saturação por bases para $60 \%$.

Após a calagem, cada porção do solo foi umedecida com água deionizada, até atingir a umidade de $0,27 \mathrm{~kg} \mathrm{~kg}^{-1}$ de $\mathrm{H}_{2} \mathrm{O}$, e foi incubada até à estabilização do $\mathrm{pH}$ (25 dias). Após este período, cada porção de solo foi seca ao ar à sombra e peneirada em peneira de $4 \mathrm{~mm}$ malha, realizando-se nova análise química (Quadro 1).

A relação entre as doses de calcário e as saturações por $\mathrm{Al}(\mathrm{m})$, após a incubação, foram estabelecidas por meio da função: $\mathrm{m}=93,24-28,86{ }^{*} \mathrm{X}+2,07^{* *} \mathrm{X}^{2} \mathrm{R}^{2}=$ $0,99\left({ }^{* *},{ }^{*}\right.$ significativos a 1 e $5 \%$, respectivamente), em que X representa a dose de calcário em $\mathrm{t}$ ha-1.

As porções de solo correspondentes aos anéis superiores receberam adubação com os seguintes nutrientes, em mg kg-1: P - 434; K - 150; N - 50; S 40; B - 0,81; Cu - 1,33; Fe - 1,55; Mn - 3,66; Mo - 0,15; e $\mathrm{Zn}$ - 4,0. Estes nutrientes foram, respectivamente, adicionados nas formas de $\mathrm{K}_{2} \mathrm{SO}_{4}, \mathrm{KH}_{2} \mathrm{PO}_{4}$, $\mathrm{NH}_{4} \mathrm{H}_{2} \mathrm{PO}_{4}, \mathrm{CaHPO}_{4}, \mathrm{H}_{3} \mathrm{BO}_{3}, \mathrm{CuSO}_{4} .5 \mathrm{H}_{2} \mathrm{O}$, $\mathrm{FeCl}_{3} .6 \mathrm{H}_{2} \mathrm{O}, \mathrm{MnCl}_{2} .4 \mathrm{H}_{2} \mathrm{O}, \mathrm{NaMoO}_{4} \cdot 2 \mathrm{H}_{2} \mathrm{O}$ e $\mathrm{ZnSO}_{4} .7 \mathrm{H}_{2} \mathrm{O}$.

Quadro 1. Caracterização química das amostras de solo após a incubação com doses de calcário correspondentes a 0,$0 ; 0,1 ; 0,37 ; 0,64 ; 0,9 ; 1,45$ e 2,0 vezes a necessidade de calagem para os anéis inferiores (intermediário+inferior) e 1,9 vezes a necessidade de calagem para o anel superior, expressa em $\mathrm{t} \mathrm{ha}^{-1}$

\begin{tabular}{|c|c|c|c|c|c|c|c|c|}
\hline \multirow{3}{*}{ Característica } & \multicolumn{8}{|c|}{ Dose de calcário $\left(\mathrm{t} \mathrm{ha}^{-1}\right)$} \\
\hline & \multicolumn{8}{|c|}{ Anel superior } \\
\hline & $\mathbf{0 , 0}$ & 0,49 & 1,7 & 2,9 & 4,1 & 6,6 & 9,3 & 8,8 \\
\hline $\mathrm{m}(\%)$ & 93,3 & 79,5 & 50,4 & 26,8 & 9,4 & 0,0 & 0,0 & 0,0 \\
\hline Carbono (dag kg-1) & 3,5 & 3,4 & 3,5 & 3,2 & 3,4 & 3,2 & 3,1 & 3,1 \\
\hline $\mathrm{P}\left(\mathrm{mg} \mathrm{dm}^{-3}\right)^{(1)}$ & 2,8 & 2,6 & 2,7 & 2,5 & 2,7 & 2,7 & 2,6 & 2,5 \\
\hline $\mathrm{K}(\mathrm{mg} \mathrm{dm}-3)^{(1)}$ & 8,0 & 9,0 & 7,0 & 10,0 & 9,0 & 7,0 & 8,0 & 9,0 \\
\hline $\mathrm{pH}\left(\mathrm{H}_{2} \mathrm{O} \quad 1: 25\right)$ & 3,9 & 4,1 & 4,4 & 4,7 & 5,0 & 5,6 & 6,2 & 5,9 \\
\hline $\mathrm{Ca}\left(\mathrm{cmol}_{\mathrm{c}} \mathrm{dm}^{-3}\right)^{(2)}$ & 0,22 & 0,57 & 1,41 & 2,28 & 3,07 & 4,81 & 6,71 & 4,1 \\
\hline $\mathrm{Mg}\left(\mathrm{cmol}_{\mathrm{c}} \mathrm{dm}^{-3}\right)^{(2)}$ & 0,44 & 0,09 & 0,25 & 0,42 & 0,59 & 0,96 & 1,30 & 1,0 \\
\hline $\mathrm{SB}\left(\mathrm{cmol}_{\mathrm{c}} \mathrm{dm}^{-3}\right)$ & 0,3 & 0,7 & 1,7 & 2,7 & 3,8 & 5,8 & 8,1 & 5,1 \\
\hline $\mathrm{H}+\mathrm{Al}\left(\mathrm{cmol}_{\mathrm{c}} \mathrm{dm}^{-3}\right)^{(3)}$ & 7,5 & 7,3 & 6,7 & 6,0 & 5,7 & 4,8 & 4,0 & 5,4 \\
\hline $\mathrm{V}(\%)$ & 6,6 & 9,1 & 18,6 & 27,3 & 35,9 & 53,0 & 72,2 & 49,0 \\
\hline Acidez trocável $\left(\mathrm{cmol}_{\mathrm{c}} \mathrm{dm}^{-3}\right)^{(2)}$ & 2,2 & 2,0 & 1,7 & 1,3 & 1,0 & 0,25 & 0,0 & 0,0 \\
\hline Al trocável $\left(\mathrm{cmol}_{\mathrm{c}} \mathrm{dm}^{-3}\right)^{(2)},(4)$ & 1,5 & 1,0 & 0,6 & 0,3 & 0,2 & 0,02 & 0,0 & 0,0 \\
\hline
\end{tabular}

$\overline{(1)}$ Extrator Mehlich-1 (Vettori, 1969). ${ }^{(2)}$ Extrator KCl $1 \mathrm{~mol} \mathrm{~L}^{-1}$ (Vettori, 1969). ${ }^{(3)}$ Extrator Ca(OAc) 20,5 mol L ${ }^{-1} \mathrm{pH}_{7,0 .}{ }^{(4)} \mathrm{Método}$ do Aluminon. 
Como variedade tolerante, utilizou-se a Icatu 4045 (cruzamento intra-específico de $C$. canephora e $C$. arabica), de sementes provenientes da fazenda experimental da EPAMIG - Patrocínio, MG. A variedade sensível utilizada foi a linhagem Catuaí 2147-H-2077-2-5-99 (cruzamento interespecífico de $C$. arabica), de sementes provenientes de Viçosa, MG. Ambas as variedades foram selecionadas por Braccini et al. (1996) quanto à tolerância ao Al.

Plântulas de café recém-germinadas em rolo de papel foram selecionadas no estádio de palito de fósforo, sendo transferidas duas plântulas por vaso. A umidade do solo foi mantida próxima a $0,27 \mathrm{~kg} \mathrm{~kg}^{-1}$ de $\mathrm{H}_{2} 0$, por meio de irrigações diárias.

As plantas foram colhidas aos 6,5 meses após o plantio nos vasos. As folhas superiores (FS) (folhas da porção superior de cada galho), as folhas inferiores (FI) e os caules (C) foram lavados por imersão rápida em água deionizada, secos em papel-toalha e acondicionados separadamente em sacos de papel. As raízes das plantas foram divididas por anéis e, posteriormente, separadas do solo por peneiramento em peneira de $2 \mathrm{~mm}$ de malha, catação manual e lavagem com jatos de água.

As raízes, de cada anel, e a parte aérea foram secas separadamente em estufa de circulação forçada de ar, a $70^{\circ} \mathrm{C}$, por $72 \mathrm{~h}$, para determinação do peso da matéria seca e, posteriormente, moídas em moinho tipo Wiley, com peneira de 20 malhas por polegada quadrada. Amostras da parte aérea e das raízes foram submetidas à digestão nítrico-perclórica (proporção 4:1) (Ganje \& Page, 1974) e analisadas quanto aos teores de $\mathrm{Ca}, \mathrm{Mg}, \mathrm{P}$ e $\mathrm{Al}$. Os teores de $\mathrm{Ca}, \mathrm{Mg}, \mathrm{Al}$ foram determinados por espectrofotometria de absorção atômica e o de $\mathrm{P}$, por colorimetria, pelo método do azul de molibdênio (Braga \& Defelipo, 1974). A eficiência de utilização dos nutrientes (EFU) foi calculada com base na seguinte equação: $\mathrm{EFU}=\left[(\text { matéria seca })^{2} /\right.$ (conteúdo do nutriente)] (Siddiqui \& Glass, 1981).

Os dados foram submetidos à análise de variância e regressão, usando-se o software SAEG (Euclydes, 1983). A escolha das equações de regressão foi realizada, inicialmente, com base no coeficiente de determinação $\left(\mathrm{R}^{2}\right)$, descartando-se aqueles com $\mathrm{R}^{2}$ inferior a $50 \%$. Em seguida, testaram-se os coeficientes dos modelos com base no quadrado médio do resíduo da análise de variância, até $10 \%$ de probabilidade pelo teste $\mathrm{F}\left(\mathrm{F}=\left[\left(\mathrm{t}^{2 *} \mathrm{Quadrado}\right.\right.\right.$ Médio do Resíduo Independente da Regressão)/(Quadrado Médio do Resíduo da ANOVA/Número de Repetições)]. Finalmente, para dois ou mais modelos com coeficientes significativos, a seleção foi feita com base no maior $\mathrm{R}^{2}$ ajustado.

\section{RESULTADOS E DISCUSSÃO}

O teor de $\mathrm{Al}$ na parte aérea da variedade Catuaí não variou com a calagem, apresentando média de
$184 \mathrm{mg} \mathrm{kg}^{-1}$ de Al (Quadro 2). Sem a calagem subsuperficial $(\mathrm{m}=93 \%)$, a variedade Icatu apresentou maior teor de $\mathrm{Al}$ na parte aérea (207 mg kg-1 que a Catuaí, $184 \mathrm{mg} \mathrm{kg}^{-1}$, quadro 2 e figura 1a). Aplicação de 4,1 t ha-1 de calcário ao solo subsuperficial reduziu o teor de $\mathrm{Al}$ em $29 \%$ (175 mg kg-1) na variedade Icatu, ficando este abaixo do valor de $184 \mathrm{mg} \mathrm{kg}^{-1}$ verificado para Catuaí. Nas raízes, a resposta foi inversa entre as variedades, a Icatu (Quadro 2) não apresentou variações no teor de Al com a aplicação de calcário na subsuperfície (3.173 mg kg-1), enquanto, para a Catuaí, observouse decréscimo linear no teor de $\mathrm{Al}$ das raízes, que atingiu um máximo de $27 \%$ com a aplicação de $9 \mathrm{t} \mathrm{ha}^{-1}$ de calcário, $4.214 \mathrm{mg} \mathrm{kg}^{-1}$ para $3.093 \mathrm{mg} \mathrm{kg}^{-1}$ (Quadro 2 e Figura 1b).

Em ambas as variedades, as raízes apresentaram maior teor de $\mathrm{Al}$ em comparação com a parte aérea (Quadro 2 e Figura 1a e 1b) em todas as doses de calcário. Respostas semelhantes foram observadas em cafeeiro por Braccini (1995), Martinez \& Monnerat (1991) e Mattiello et al. (2004), concordando com as do trabalho de Wagatsunaga (1984), que afirma ser o Al elemento de baixa mobilidade na planta, sendo preferencialmente acumulado nas raízes. Rodrigues et al. (2001) observaram maiores sintomas de toxidez de $\mathrm{Al}$ nas raízes da variedade Catuaí em relação à variedade Icatu, decorrentes, provavelmente, do maior acúmulo do elemento nessa parte da planta, como foi comprovado no presente trabalho.

$\mathrm{Na}$ parte aérea, somente foram observadas variações no teor de Al nas folhas superiores da variedade Icatu (Quadro 2 e Figura 1c). Nas folhas inferiores e nos caules de ambas as variedades, não foram observadas variações na alocação de $\mathrm{Al}$ em função da calagem na subsuperfície (Quadro 2). Os teores de $\mathrm{Al}$ nas folhas superiores da variedade Icatu diminuíram de 216 para $161 \mathrm{mg} \mathrm{kg}^{-1}$ com a aplicação de 4,8 $\mathrm{t}$ ha-1 de calcário no solo (Quadro 2 e Figura 1c), enquanto, na variedade Catuaí, o teor médio de $\mathrm{Al}$ nas folhas superiores manteve-se em $181 \mathrm{mg} \mathrm{kg}^{-1}$ (Quadro 2).

Pavan et al. (1982), cultivando cafeeiro até sete meses em solo com alta atividade de $\mathrm{Al}$ e devidamente adubado, observaram valor máximo de $225 \mathrm{mg} \mathrm{kg}-1$ de $\mathrm{Al}$ no terceiro par de folhas (correspondente às folhas superiores no presente experimento), sendo este teor de $\mathrm{Al}$ associado a sintomas de toxidez. De acordo com os autores, 100 e $62 \mathrm{mg} \mathrm{kg}^{-1}$ de $\mathrm{Al}$ nas folhas parecem ser as concentrações limite, a partir das quais, respectivamente, o crescimento da parte aérea e das raízes é limitado. No presente trabalho, foi observado teor de até $263 \mathrm{mg} \mathrm{kg}^{-1}$ de $\mathrm{Al}$ nas folhas superiores e não foram observados sintomas de toxidez por Al na parte aérea.

$\mathrm{O}$ teor de $\mathrm{Al}$ nas raízes crescidas no anel superior da coluna de PVC (Quadro 2 e Figura 1d) foi menor que o teor das raízes dos demais anéis (Quadro 2), independentemente da dose de calcário aplicada ao solo nos anéis inferiores, para ambas as variedades. 
Quadro 2. Equações de regressão para teores de Alumínio $\left(\mathrm{mg} \mathrm{kg}^{-1}\right)$ na parte aérea e raízes de duas variedades de cafeeiro, considerando as doses de calcário aplicadas $\left(\mathrm{t} \mathrm{ha}^{-1}\right)$ na subsuperfície do solo

\begin{tabular}{|c|c|c|c|}
\hline Fração & Cafeeiro & Equação de regressão & $\mathbf{R}^{2}$ \\
\hline Total & $\begin{array}{l}\text { Icatu } \\
\text { Catuaí }\end{array}$ & $\begin{array}{l}\text { Parte aérea } \\
\hat{Y}=207-31,77^{\circ} \mathrm{X}^{1 / 2^{*}}+7,85^{\mathrm{ns} X} \\
\hat{Y}=\bar{Y}=184\end{array}$ & 0,80 \\
\hline Folhas superiores & $\begin{array}{l}\text { Icatu } \\
\text { Catuaí }\end{array}$ & $\begin{array}{l}\hat{Y}=216-50,44^{*} \mathrm{X}^{1 / 2^{*}}+11,65^{\mathrm{ns} \mathrm{X}} \\
\hat{Y}=\bar{Y}=181\end{array}$ & 0,70 \\
\hline $\begin{array}{l}\text { Folhas inferiores } \\
\text { Caule }\end{array}$ & $\begin{array}{l}\text { Icatu } \\
\text { Catuaí } \\
\text { Icatu } \\
\text { Catuaí }\end{array}$ & $\begin{array}{l}\hat{Y}=\bar{Y}=194 \\
\hat{Y}=\bar{Y}=199 \\
\hat{Y}=\bar{Y}=191 \\
\hat{Y}=\bar{Y}=171\end{array}$ & \\
\hline Total & $\begin{array}{l}\text { Icatu } \\
\text { Catuaí }\end{array}$ & $\begin{array}{l}\text { Raízes } \\
\hat{Y}=\bar{Y}=3173 \\
\hat{Y}=4214-124,482^{* *} \mathrm{X}\end{array}$ & 0,69 \\
\hline Anel superior & $\begin{array}{l}\text { Icatu } \\
\text { Catuaí }\end{array}$ & $\begin{array}{l}\hat{Y}=2756-863,67^{*} \times 1 / 2+294,78^{*} \mathrm{x} \\
\hat{Y}=\bar{Y}=2770\end{array}$ & 0,70 \\
\hline Anel inferior & $\begin{array}{l}\text { Icatu } \\
\text { Catuaí } \\
\text { Icatu } \\
\text { Catuaí }\end{array}$ & $\begin{array}{l}\hat{Y}=\bar{Y}=3780 \\
\hat{Y}=4843-172,75^{* *} \mathrm{X} \\
\hat{Y}=\bar{Y}=3970 \\
\hat{Y}=\bar{Y}=4211\end{array}$ & 0,69 \\
\hline
\end{tabular}

$\mathrm{ns},{ }^{* *}, * \mathrm{e}^{\circ}$, não-significativos (até $10 \%$ ), significativos a 1,5 e a $10 \%$ pelo teste $\mathrm{F}$.

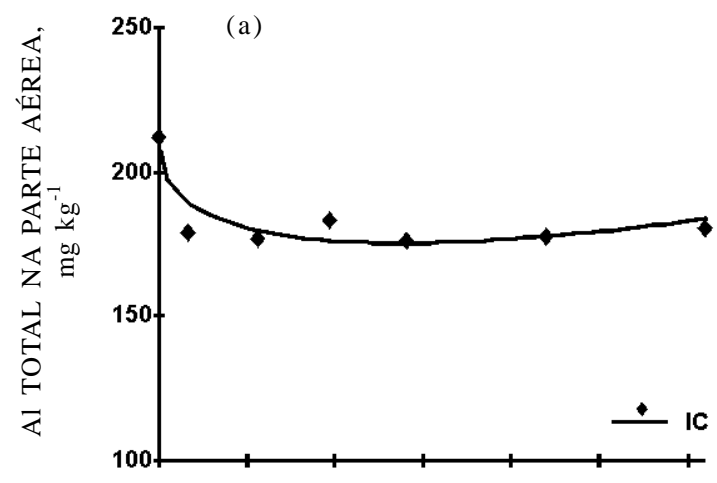

(c)

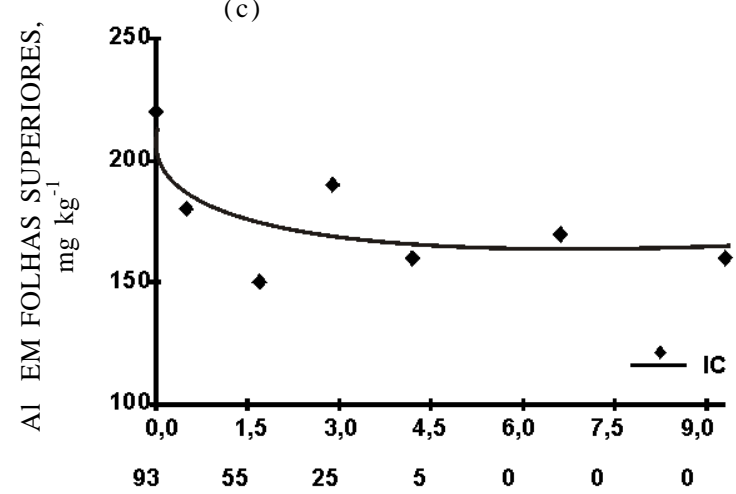

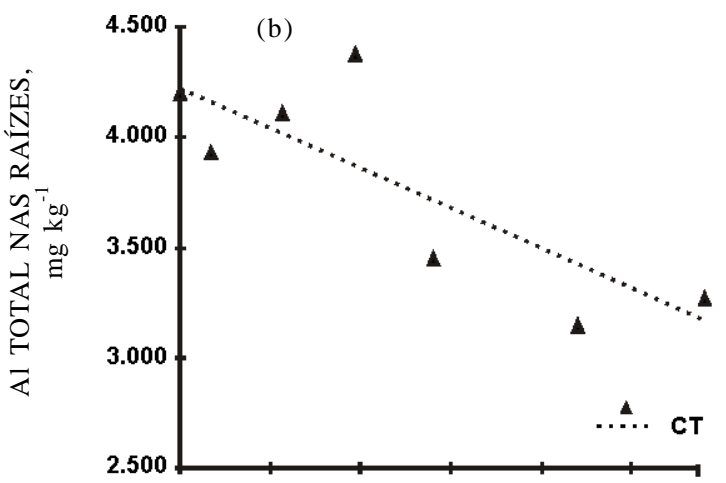

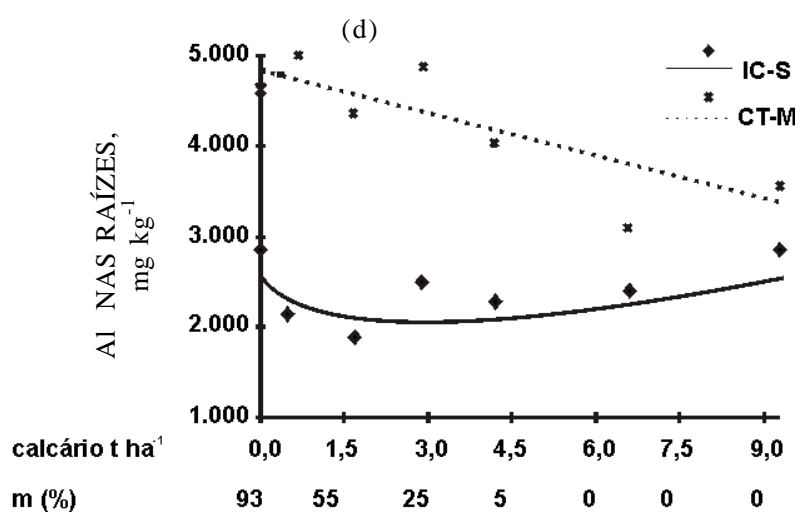

Figura 1. Teores de Al na parte aérea e raízes de duas variedades de cafeeiro, Icatu (IC) e Catuaí (CT), em função das doses de calcário aplicadas na subsuperfície do solo. a - parte aérea total; b - raiz total; c folhas superiores (FS), d - raiz do anel superior (S), raiz do anel mediano (M). 
A aplicação do calcário em subsuperfície causou decréscimo no teor de $\mathrm{Al}$ nas raízes do anel superior da variedade Icatu (Quadro 2 e Figura 1d). Nesta variedade, não foram observadas variações no teor de $\mathrm{Al}$ nas raízes do anel mediano e inferior. Na variedade Catuaí, a aplicação do calcário na subsuperfície causou decréscimo linear de $33 \%$ no teor de $\mathrm{Al}$, somente nas raízes do anel mediano. Na variedade Catuaí, a princípio, a absorção de $\mathrm{Al}$ parece ter sido relacionada com a saturação desse elemento encontrada no solo. $\mathrm{O}$ ajuste ao estresse permitiu que houvesse restrição da absorção, estabilizando-se os teores de $\mathrm{Al}$ das raízes do anel inferior. Isso indica a ação de um controle ativado ao contato das células da coifa com altos teores de $\mathrm{Al}$ no solo. Para a variedade Icatu, aliada aos mecanismos de exclusão, percebe-se certa tolerância interna, uma vez que a integridade das raízes foi mantida com transporte de $\mathrm{Al}$ para a parte aérea nas maiores saturações por $\mathrm{Al}$.

Observou-se que a aplicação do calcário em subsuperfície resultou em aumentos de 18,0 e 18,3\% nos teores de $\mathrm{P}$ nas folhas superiores nas variedades Icatu e Catuaí, respectivamente (Quadro 3 e Figura 2a), e não promoveu alteração nos teores de $\mathrm{P}$ nas folhas inferiores e no caule (Quadro 3). Martinez \& Monnerat (1991) também observaram redução no teor de P, tanto nas folhas superiores quanto nas inferiores, e no caule de cafeeiro com o aumento do $\mathrm{Al}$ em solução nutritiva, demonstrando que a maior tolerância de alguns cultivares deve-se ao melhor uso do $\mathrm{P}$, com maior translocação para regiões de metabolismo intenso.

Segundo Pavan \& Bingham (1982), o cultivo do cafeeiro na presença de $\mathrm{Al}$ causa uma precipitação interna de $\mathrm{P}$, além de o $\mathrm{Al}$ poder interferir na translocação desse elemento para a parte aérea da planta. No entanto, no presente trabalho, apesar de ter sido verificado aumento no teor de $\mathrm{P}$ nas folhas superiores com a aplicação do calcário na subsuperficie do solo, o menor teor observado nas folhas (0,197 dag kg-1 de P) mostrou-se adequado ao desenvolvimento da cultura. Para cafeeiros, os teores adequados de $\mathrm{P}$ englobam a faixa entre $0,12 \mathrm{e}$ 0,23 dag kg-1 (Malavolta 1986; Raij, 1991; Malavolta, 1992; Malavolta et al., 1997; Martinez et al., 2003). Segundo Foy (1974), muitas espécies de plantas tolerantes ao $\mathrm{Al}$ apresentam uso eficiente de $\mathrm{P}$. No presente trabalho, além de o nível de $\mathrm{P}$ estar adequado, o P absorvido foi também translocado preferencialmente para regiões de crescimento mais ativo (folhas superiores).

Os teores de $\mathrm{P}$ nas raízes dos anéis superiores foram relativamente próximos aos observados nos medianos e inferiores (variaram de 0,14 a 0,20 dag kg-1) (Quadro 3 e Figura 2b). Na variedade Catuaí, o teor de $\mathrm{P}$ nas raízes do anel mediano aumentou em cerca de $32 \%$, atingindo ponto de máximo com a aplicação de $5,2 \mathrm{t}$ ha ${ }^{-1}$ de calcário $(\mathrm{m}=0 \%)$, com posterior queda com a adição de mais calcário ao solo, sugerindo restrição na absorção de $\mathrm{P}$ em doses de calcário supra- ótimas. Nesse anel, não houve variação no teor de $\mathrm{P}$ nas raízes da variedade Icatu.

Ambas as variedades apresentaram maior teor de Ca nas folhas inferiores e menor no caule (Quadro 3). Os teores de $\mathrm{Ca}$ em folhas superiores, inferiores e caule da variedade Catuaí apresentaram incrementos de $43,3,15,5$ e $11,6 \%$, respectivamente, enquanto, para a variedade Icatu, o aumento ocorreu somente nas folhas superiores (35,5 \%) (Quadro 3 e Figura 2c). O teor de $\mathrm{Mg}$ nas folhas superiores foi maior que o das demais frações da parte aérea da planta, com acréscimos nas doses intermediárias de calcário para ambas as variedades (Quadro 3 e Figura 2e). O teor de $\mathrm{Mg}$ no caule de ambas as variedades e nas folhas inferiores da variedade Icatu apresentaram aumentos lineares com a calagem subsuperficial.

Nas raízes, verificou-se que as duas variedades apresentaram padrão semelhante no teor de Ca e de Mg em cada anel (Quadro 3 e Figura 2d e 2f). Nas raízes do anel superior de ambas as variedades, observaram-se decréscimos nos teores de $\mathrm{Ca}$ e $\mathrm{Mg}$ com a aplicação do calcário subsuperficial. As raízes do anel inferior apresentaram aumento no teor de $\mathrm{Ca}$, sendo de 128,4 e de $128,9 \%$, para as variedades Icatu e Catuaí, respectivamente, e para o $\mathrm{Mg}$ apresentaram incremento máximo de 116,6 e 135,5 \% nas respectivas variedades.

Os teores de Ca considerados normais nas folhas do cafeeiro, segundo Malavolta (1986); Malavolta (1992); Malavolta et al. (1997) e Martinez et al (2003), devem estar entre 0,84 e 1,50 dag kg-1 e para o $\mathrm{Mg}$ entre 0,29 e 0,56 dag $\mathrm{kg}^{-1}$. No presente trabalho, em todos os níveis de saturação por $\mathrm{Al}$ nos anéis inferiores, os teores de Ca e Mg nas folhas foram superiores aos citados por estes autores. A adição de Ca e de $\mathrm{Mg}$ (via calagem) no solo do anel superior manteve os teores adequados nas folhas, mesmo quando não foi aplicado calcário no solo dos anéis inferiores. Apesar dos teores adequados de Ca nas folhas, em todas as doses de calcário utilizadas, a variedade Catuaí respondeu à aplicação de calcário ao solo dos anéis inferiores, evidenciando maior sensibilidade, enquanto, para a variedade Icatu, essa resposta ocorreu somente para as folhas superiores (Quadro 3 e Figura 2c).

Nas raízes dos anéis inferiores, de ambas as variedades, foram observados incrementos acentuados no teor de Ca com pequenas doses de calcário aplicado (Quadro 3 e Figura 2d). A partir de 1,5 $\mathrm{t} \mathrm{ha}^{-1}$ de calcário aplicado no solo, os incrementos no teor de Ca nas raízes foram menos acentuados. Esta resposta indica que, a partir desta dose, a relação $\mathrm{Ca} / \mathrm{Al}$ provavelmente encontra-se em nível mais tolerável pela planta. Segundo Boudot et al. (1994), a relação $\mathrm{Ca} / \mathrm{Al}$ pode refletir a competição entre o $\mathrm{Ca}$ e o $\mathrm{Al}$ por sítios de absorção nas raízes.

Nas raízes, não houve alteração da eficiência de utilização de $\mathrm{P}$ com a aplicação do calcário ao solo para ambas as variedades (Quadro 4). Para a parte aérea da variedade Catuaí também não se observaram 
Quadro 3. Equações de regressão para teores de $\mathrm{P}$, Ca e $\mathrm{Mg}\left(\mathrm{dag}_{\mathrm{kg}}{ }^{-1}\right)$ na parte aérea e raízes de duas variedades de cafeeiro, em função das doses de calcário aplicadas ( $\mathrm{t} \mathrm{ha}^{-1}$ ) na subsuperfície do solo

\begin{tabular}{|c|c|c|c|}
\hline Fração & Cafeeiro & Equação de regressão & $\mathbf{R}^{2}$ \\
\hline & \multicolumn{3}{|c|}{ P na parte aérea } \\
\hline Folhas superiores & $\begin{array}{l}\text { Icatu } \\
\text { Catuaí }\end{array}$ & $\begin{array}{l}\hat{Y}=0,199+0,00385^{* *} \mathrm{X} \\
\hat{Y}=0,200+0,0039^{* *} \mathrm{X}\end{array}$ & $\begin{array}{l}0,65 \\
0,61\end{array}$ \\
\hline Folhas inferiores & $\begin{array}{l}\text { Icatu } \\
\text { Catuaí }\end{array}$ & $\begin{array}{l}\hat{Y}=\bar{Y}=0,197 \\
\hat{Y}=\bar{Y}=0,220\end{array}$ & \\
\hline Caule & $\begin{array}{l}\text { Icatu } \\
\text { Catuaí }\end{array}$ & $\begin{array}{l}\hat{Y}=\bar{Y}=0,105 \\
\hat{Y}=\bar{Y}=0,107\end{array}$ & \\
\hline & \multicolumn{3}{|c|}{$\mathrm{P}$ nas raízes } \\
\hline Anel superior & $\begin{array}{l}\text { Icatu } \\
\text { Catuaí }\end{array}$ & $\begin{array}{l}\hat{Y}=\bar{Y}=0,131 \\
\hat{Y}=\bar{Y}=0,145\end{array}$ & \\
\hline Anel mediano & $\begin{array}{l}\text { Icatu } \\
\text { Catuaí }\end{array}$ & $\begin{array}{l}\hat{Y}=\bar{Y}=0,176 \\
\hat{Y}=0,153+0,0189^{* *} \mathrm{X}-0,0018^{* *} \mathrm{X}^{2}\end{array}$ & 0,88 \\
\hline Aanel inferior & $\begin{array}{l}\text { Icatu } \\
\text { Catuaí }\end{array}$ & $\begin{array}{l}\hat{Y}=0,160-0,0196^{* *} \mathrm{X}+0,0059^{* *} \mathrm{X}^{2}-0,00043^{* *} \mathrm{X}^{3} \\
\hat{Y}=0,151-0,0142^{\text {ns }} \mathrm{X}+0,0044 \mathrm{X}^{2}-0,00033^{* *} \mathrm{X}^{3}\end{array}$ & $\begin{array}{l}0,96 \\
0,67\end{array}$ \\
\hline & \multicolumn{3}{|c|}{ Ca na parte aérea } \\
\hline Folhas superiores & $\begin{array}{l}\text { Icatu } \\
\text { Catuaí }\end{array}$ & $\begin{array}{l}\hat{Y}=1,133+0,043^{* *} \mathrm{X} \\
\hat{Y}=1,240+0,058^{* *} \mathrm{X}\end{array}$ & $\begin{array}{l}0,90 \\
0,95\end{array}$ \\
\hline Folhas inferiores & $\begin{array}{l}\text { Icatu } \\
\text { Catuaí }\end{array}$ & $\begin{array}{l}\hat{Y}=\bar{Y}=1,900 \\
\hat{Y}=1,910+0,032^{* *} \mathrm{X}\end{array}$ & 0,85 \\
\hline Caule & $\begin{array}{l}\text { Icatu } \\
\text { Catuaí }\end{array}$ & $\begin{array}{l}\hat{Y}=\bar{Y}=0,430 \\
\hat{Y}=0,540-0,127^{* *} \mathrm{X}^{1 / 2}+0,0656^{\mathrm{ns}} \mathrm{X}\end{array}$ & 0,95 \\
\hline & \multicolumn{3}{|c|}{ Ca nas raízes } \\
\hline Anel superior & $\begin{array}{l}\text { Icatu } \\
\text { Catuaí }\end{array}$ & $\begin{array}{l}\hat{Y}=0,615-0,131^{*} \mathrm{X}^{1 / 2}+0,036^{\mathrm{ns}} \mathrm{X} \\
\hat{Y}=0,786-0,19^{* *} \mathrm{X}^{1 / 2}+0,043^{*} \mathrm{X}\end{array}$ & $\begin{array}{l}0,87 \\
0,62\end{array}$ \\
\hline Anel mediano & $\begin{array}{l}\text { Icatu } \\
\text { Catuaí }\end{array}$ & $\begin{array}{l}\hat{Y}=0,601+0,06^{* *} \mathrm{X} \\
\hat{Y}=0,712+0,047^{* *} \mathrm{X}\end{array}$ & $\begin{array}{l}0,80 \\
0,86\end{array}$ \\
\hline Anel inferior & $\begin{array}{l}\text { Icatu } \\
\text { Catuaí }\end{array}$ & $\begin{array}{l}\hat{Y}=0,900+0,52^{* *} \mathrm{X}^{1 / 2}-0,045^{\mathrm{ns}} \mathrm{X} \\
\hat{Y}=0,953+0,959^{* *} \mathrm{X}^{1 / 2}-0,187^{* *} \mathrm{X}\end{array}$ & $\begin{array}{l}0,85 \\
0,89\end{array}$ \\
\hline & \multicolumn{3}{|c|}{ Mg na parte aérea } \\
\hline Folhas superiores & $\begin{array}{l}\text { Icatu } \\
\text { Catuaí }\end{array}$ & $\begin{array}{l}\hat{Y}=0,339+0,0852^{* *} \mathrm{X}^{1 / 2}-0,01648^{*} \mathrm{X} \\
\hat{Y}=0,384+0,036^{* *} \mathrm{X}-0,0035^{* *} \mathrm{X}^{2}\end{array}$ & $\begin{array}{l}0,66 \\
0,87\end{array}$ \\
\hline Folhas inferiores & $\begin{array}{l}\text { Icatu } \\
\text { Catuaí }\end{array}$ & $\begin{array}{l}\hat{Y}=\bar{Y}=0,286 \\
\hat{Y}=0,265+0,0149^{* *} \mathrm{X}\end{array}$ & 0,98 \\
\hline Caule & $\begin{array}{l}\text { Icatu } \\
\text { Catuaí }\end{array}$ & $\begin{array}{l}\hat{Y}=0,141+0,0047^{*} \mathrm{X} \\
\hat{Y}=0,213+0,008^{* *} \mathrm{X}\end{array}$ & $\begin{array}{l}0,64 \\
0,81\end{array}$ \\
\hline & \multicolumn{3}{|c|}{ Mg nas raízes } \\
\hline Anel superior & $\begin{array}{l}\text { Icatu } \\
\text { Catuaí }\end{array}$ & $\begin{array}{l}\hat{Y}=0,479-0,1011^{*} \mathrm{X}^{1 / 2}+0,0235^{\mathrm{ns}} \mathrm{X} \\
\hat{Y}=0,562-0,089^{*} \mathrm{X}+0,008^{* *} \mathrm{X}^{2}\end{array}$ & $\begin{array}{l}0,66 \\
0,95\end{array}$ \\
\hline Anel mediano & $\begin{array}{l}\text { Icatu } \\
\text { Catuaí }\end{array}$ & $\begin{array}{l}\hat{Y}=0,760-0,0164^{\mathrm{ns} X}+0,0072^{* *} \mathrm{X}^{2} \\
\hat{Y}=0,640+0,039^{* *} \mathrm{X}\end{array}$ & $\begin{array}{l}0,91 \\
0,94\end{array}$ \\
\hline Anel inferior & $\begin{array}{l}\text { Icatu } \\
\text { Catuaí }\end{array}$ & $\begin{array}{l}\hat{Y}=0,740+0,829^{* *} \mathrm{X}^{1 / 2}-0,197^{* *} \mathrm{X} \\
\hat{Y}=0,693+0,913^{* *} \mathrm{X}^{1 / 2}-0,22^{* *} \mathrm{X}\end{array}$ & $\begin{array}{l}0,94 \\
0,93\end{array}$ \\
\hline
\end{tabular}

ns, ** e *, não-significativo (até $5 \%$ ), significativos a 1 e a $5 \%$ pelo teste $\mathrm{F}$. 
(a)
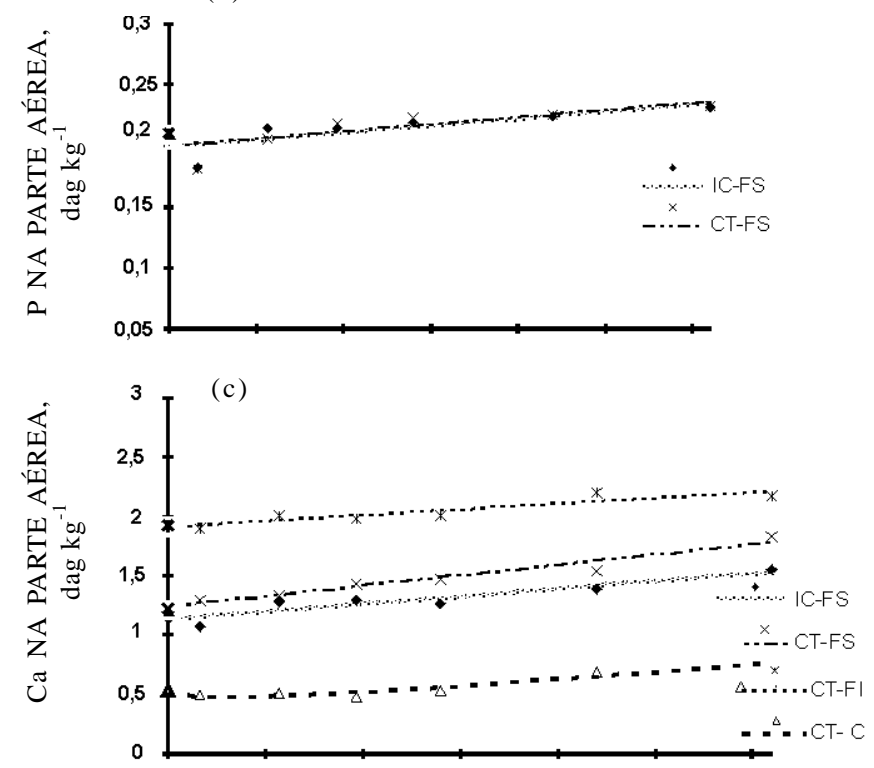

(e)

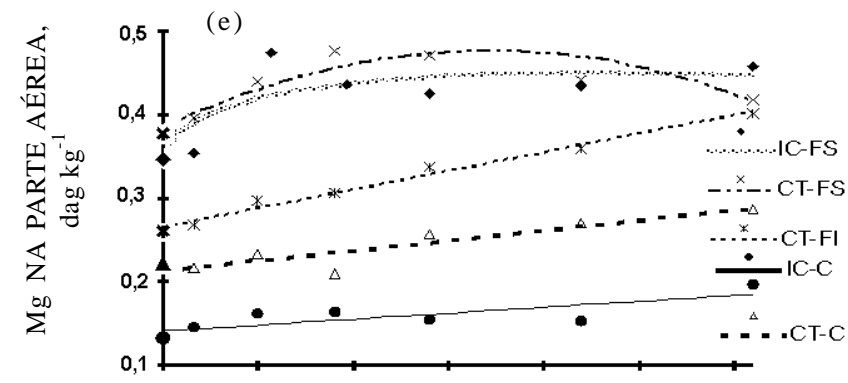

(g)

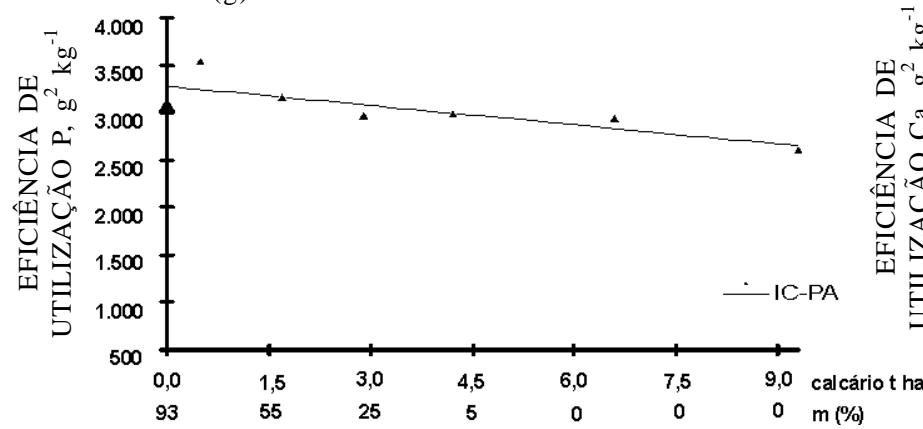

(b)
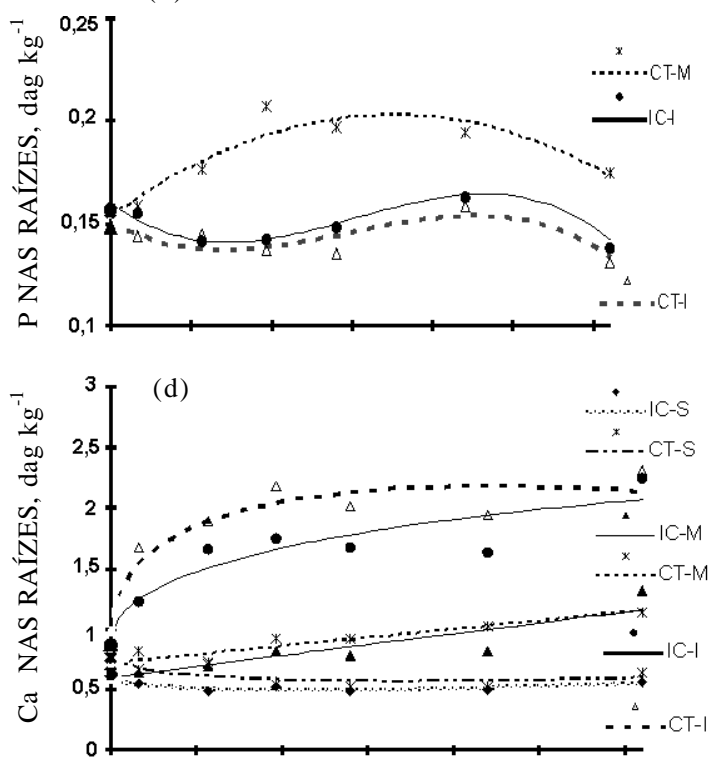

(f)

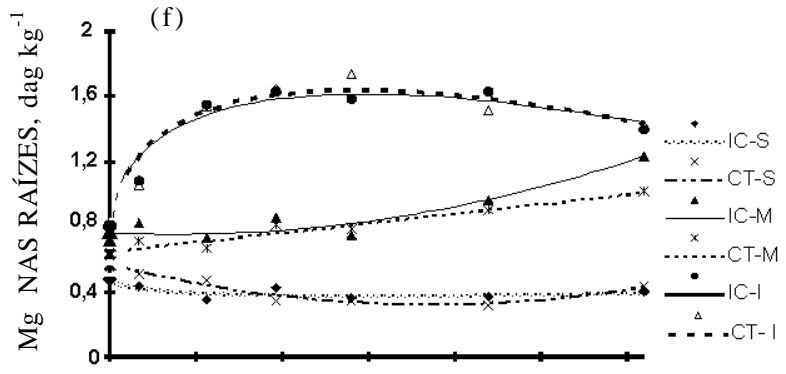

(h)

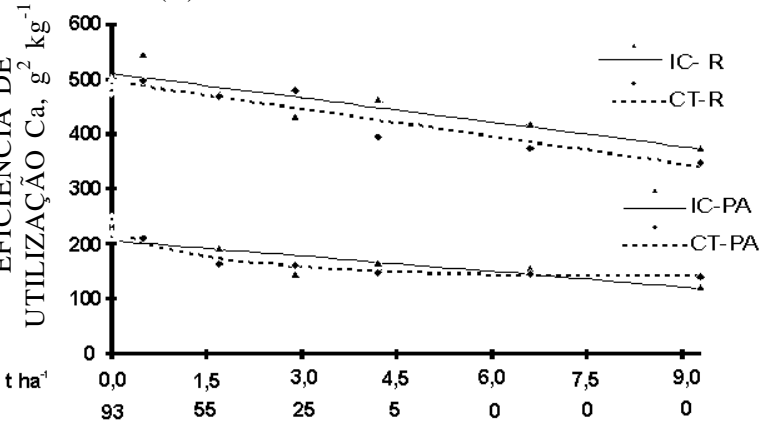

Figura 2. Teores na parte aérea e nas raízes de $\mathbf{P}(\mathrm{a}, \mathrm{b})$, de Ca (c, d) e de $\mathrm{Mg}(\mathrm{e}, \mathrm{f})$ e eficiência de utilização de P (g) e Ca (h) de duas variedades de cafeeiro, Icatu (IC) e Catuaí (CT), em função das doses de calcário aplicadas na subsuperfície do solo. Folhas superiores (FS), folhas inferiores (FI), caule (C), raiz do anel superior (S), raiz do anel mediano (M), raiz do anel inferior (I), raízes totais (R) e parte aérea total (PA).

variações na eficiência de utilização de P (Quadro 4), enquanto, para a Icatu, observou-se decréscimo de 19,3 \% nessa característica com a aplicação do calcário no solo (Quadro 4 e Figura 2g). Este decréscimo da eficiência de utilização de $\mathrm{P}$ coincide com o decréscimo no teor de $\mathrm{Al}$ (Figura 1a) e com o aumento do teor de $\mathrm{P}$ nas folhas superiores (Figura 2a), em função da aplicação do calcário na subsuperfície. Tal resultado indica que, em maiores saturações por Al na subsuperfície do solo, a planta apresenta menor teor de $\mathrm{P}$, por outro lado, aumenta a eficiência de utilização deste elemento para manter a produção de matéria seca estável. Segundo Raju et al. (1987), plantas com boa eficiência de utilização de $\mathrm{P}$ apresentam maior taxa de retranslocação, ou seja, maior redistribuição do $\mathrm{P}$ de tecidos velhos para os mais novos.

Comparando as respostas das duas variedades testadas, pode-se pressupor que, pelo menos no que se refere à parte aérea, a maior eficiência de utilização de $\mathrm{P}$ está associada ao acúmulo de $\mathrm{Al}$ nessa parte da 
Quadro 4. Equações de regressão para eficiência de utilização de Ca e $P\left(g^{2} \mathbf{k g}^{-1}\right)$ na parte aérea e raízes de duas variedades de cafeeiro, considerando as doses de calcário aplicadas (t ha $\left.\mathbf{~ h}^{-1}\right)$ na subsuperfície do solo

\begin{tabular}{|c|c|c|c|}
\hline Fração & Cafeeiro & Equação de regressão & $\mathbf{R}^{2}$ \\
\hline & & Eficiencia de utilização de $\mathrm{P}$ & \\
\hline \multirow[t]{2}{*}{ Parte aérea } & Icatu & $\hat{Y}=3.279-67,93^{*} \mathrm{X}$ & 0,67 \\
\hline & Catuaí & $\hat{Y}=\bar{Y}=3.172$ & \\
\hline \multirow[t]{3}{*}{ Raízes } & Icatu & $\hat{Y}=\bar{Y}=1.057$ & \\
\hline & Catuaí & $\hat{Y}=\bar{Y}=1.294$ & \\
\hline & & Eficiencia de utilização de $\mathrm{Ca}$ & \\
\hline \multirow[t]{2}{*}{ Parte aérea } & Icatu & $\hat{Y}=510-14,79^{* *} \mathrm{X}$ & 0,81 \\
\hline & Catuaí & $\hat{Y}=494-16,59^{* *} \mathrm{X}$ & 0,88 \\
\hline \multirow[t]{2}{*}{ Raízes } & Icatu & $\hat{Y}=206-6,33^{*} \mathrm{X}$ & 0,80 \\
\hline & Catuaí & $\hat{Y}=248-74,71^{* *} \mathrm{X}^{1 / 2}+13,08^{\mathrm{ns} X}$ & 0,98 \\
\hline
\end{tabular}

ns, ** e *, não-significativo (até $5 \%$ ), significativos a 1 e a $5 \%$ pelo teste $\mathrm{F}$.

planta. Esse resultado também foi observado por Braccini (1995, 2000), que constatou que a tolerância ao $\mathrm{Al}$ de certas variedades de cafeeiro cultivadas em solução nutritiva, em presença de Al, estava associada à eficiência de utilização de $\mathrm{P}$ pelas plantas.

A eficiência de utilização de Ca na parte aérea e nas raízes decresceu com a aplicação do calcário no solo para as duas variedades (Quadro 4 e Figura 2h). Na parte aérea, a redução foi de 27,0 e 31,2 \% e, nas raízes, de 42,3 e 43,5\%, para as variedades Icatu e Catuaí, respectivamente. Esse decréscimo deveu-se ao aumento no teor de Ca tanto na parte aérea quanto nas raízes, sem, entretanto, promover alteração na produção de matéria seca nestas partes com a aplicação do calcário ao solo dos anéis inferiores (Rodrigues et al., 2001). Assim, a maior absorção de Ca não implicou maior produção de biomassa. Esse resultado está de acordo com os dados obtidos por Braccini (1995), que observou que a toxidez ao $\mathrm{Al}$ implicou o aumento da eficiência de utilização de Ca, principalmente nos genótipos mais tolerantes ao $\mathrm{Al}$. Os genótipos tolerantes ao $\mathrm{Al}$ seriam aqueles com maior capacidade de produção de biomassa, mesmo com redução na absorção de Ca.

Variações nos padrões de distribuição de raízes no perfil do solo com a calagem foram observadas por Rodrigues et al. (2001). Os autores verificaram que a calagem nos anéis inferiores proporcionou maior percentual de matéria seca, comprimento e superfície de raízes nesses anéis em relação ao percentual de raízes no anel superior, independentemente de serem as plantas sensíveis ou tolerantes ao Al. Assim, em situação de alto teor de Al na subsuperfície, proporcionalmente, maiores quantidades de raízes são alocadas para a camada do solo corrigida (anel superior) em relação às camadas do solo não corrigidas (anel inferior). Este ajuste na alocação de raízes no perfil do solo, em função do teor de $\mathrm{Al}$ na subsuperfície observado pelos autores, provavelmente, foi importante para manter adequados os teores de nutrientes na planta, conforme verificado neste trabalho. Com isso, as plantas de cafeeiro cultivadas em solo com a camada de 0-12 cm corrigida, adubada e sem restrição hídrica não tiveram problemas nutricionais quanto à carência de $\mathrm{P}$, Ca e Mg, ou toxidez de $\mathrm{Al}$, independentemente do teor de $\mathrm{Al}$ encontrado na subsuperfície.

\section{CONCLUSÕES}

1. As variedades Catuaí (mais sensível) e Icatu (mais tolerante) apresentaram diferentes mecanismos de ajuste ao estresse por Al. Catuaí manteve inalteradas as concentrações de $\mathrm{Al}$ da parte aérea, enquanto as concentrações radiculares aumentaram com saturações crescentes por $\mathrm{Al}$ em subsuperfície, sugerindo a atuação de um mecanismo de exclusão ou compartimentalização do elemento nas raízes. Icatu manteve inalteradas e mais baixas as concentrações radiculares de $\mathrm{Al}$, o que previne a toxidez radicular do elemento, transportando-o para a parte aérea, sugerindo, além de mecanismos de exclusão ou compartimentalização, a atuação de algum mecanismo de tolerância interna ao elemento.

2. A aplicação de calcário em subsuperfície aumentou os teores de Ca e Mg e diminuiu a eficiência de utilização de Ca pela parte aérea e pelas raízes em ambas as variedades de cafeeiro testadas.

3. A correção do solo na subsuperfície aumentou o teor de $\mathrm{P}$ de folhas superiores e diminuiu o teor de $\mathrm{Al}$ e a eficiência de utilização de $\mathrm{P}$ e de $\mathrm{Ca}$ pela parte aérea da variedade Icatu (tolerante). 
4. Para plantas com até 6,5 meses de idade, a adubação e a correção da acidez da camada superficial do solo foram eficientes para manter os teores de $\mathrm{P}$, $\mathrm{Ca}$ e $\mathrm{Mg}$ em níveis considerados adequados para o cafeeiro, tanto para a variedade sensível quanto para a variedade tolerante ao $\mathrm{Al}$, em solo com até $90 \%$ de saturação por $\mathrm{Al}$ na subsuperfície.

\section{LITERATURA CITADA}

BOUDOT, J.P.; BECQUER, T.; MERLET, D. \& ROUELLER, J. Aluminium toxicity en declining forest: a general overview with a seasonal assessment in a silver fir forest in the Vosges Moutains (France). Ann. Sci. For., 51:27. 51,1994 .

BRACCINI, M.C.L. Comportamento de nove populações de café quanto à tolerância ao alumínio em solução nutritiva. Viçosa, MG, Universidade Federal de Viçosa, 1995. 92p. (Tese de Mestrado)

BRACCINI, M.C.L. Tolerância ao Al por genótipos de cafeeiro em solução nutritiva e no solo. Viçosa, MG, Universidade Federal de Viçosa, 2000. 102p. (Tese de Doutorado)

BRACCINI, M.C.L.; MARTINEZ, H.E.P.; PEREIRA, P.R.G. \& SAMPAIO, N.F. Tolerância de genótipos de cafeeiro ao alumínio em solução nutritiva. I Crescimento e desenvolvimento da parte aérea e sistema radicular. $\mathrm{R}$. Bras. Ci. Solo, 22:435-442, 1998a.

BRACCINI, M.C.L.; MARTINEZ, H.E.P.; PEREIRA, P.R.G.; SAMPAIO, N.F. \& SILVA, E.A.M. Aluminium tolerance of coffee populations. In: INTERNATIONAL SYMPOSIUM ON PLANT-SOIL INTERACTIONS AT LOW pH, 4., Belo Horizonte, 1996. Resumos. Belo Horizonte, 1996.

BRACCINI, M.C.L.; MARTINEZ, H.E.P.; PEREIRA, P.R.G.; SAMPAIO, N.F. \& PEREIRA, A.A. Tolerância de genótipos de cafeeiro ao alumínio em solução nutritiva. II Teores de $\mathrm{P}, \mathrm{Ca}$, e Al e eficiência ao P e Ca. R. Bras. Ci. Solo, 22:443$450,1998 \mathrm{~b}$

BRAGA, J.M. \& DEFELIPO, B.V. Determinação espectrofotométrica do fósforo com extrato de solos e plantas. R. Ceres, 21:73-85, 1974.

CLARKSON, D.T. Effect of aluminum on growth and mineral elements of Al-tolerant and barley seedling. Plant Physiol., 41:165-172, 1966.

EUCLYDES, R.F. Sistema para análise estatísticas e genéticas (SAEG) - manual provisório. Viçosa, MG, Universidade Federal de Viçosa, 1983. 74p.

FOY, D.C. Effects of aluminum on plant growth. In: CARSON, E.W., ed. The plant root and its environment. Charlottesville, University Press of Virginia, 1974. p.601642 .

FURLANI, P.R. Efeitos fisiológicos do alumínio em plantas. In: SIMPÓSIO AVANÇADO DE SOLOS E NUTRIÇÃO DE PlANTAS, 2; Piracicaba, 1989. Anais. Piracicaba, Escola Superior de Agricultura "Luiz de Queiroz", 1989. p.73-92.
GANJE, T.J. \& PAGE, A.L. Rapid dissolution of tissue for cadmium determination by atomic absorption espectrophotometry. Atmos. Absor. News, 13:131-134, 1974 .

GUIMARÃES, P.T.G. \& LOPES, A.S. Solos para o cafeeiro: características, propriedades e manejo. In: RENA, A.B.; MALAVOLTA, E.; ROCHA. M. \& YAMADA, T., eds Cultura do cafeeiro, fatores que afetam a produtividade. Piracicaba, POTAFOS. 1986. p.115-149.

HUANG, J.W.; SHAFF, J.E.; GRUNES, D.L. \& KOCHIAN, L.V. Aluminum effects on calcium fluxes at the root apex of aluminum-tolerant and aluminum-sensitive wheat cultivars. Plant Physiol., 98:230-237, 1992.

MALAVOLTA, E. ABC da análise de solo e de folhas. São Paulo, Agronômica Ceres, 1992. 124p.

MALAVOLTA, E. Nutrição, adubação e calagem para o cafeeiro. In: RENA, A.B.; MALAVOLTA, E.; ROCHA, M. \& YAMADA, T., eds. Cultura do cafeeiro, fatores que afetam a produtividade. Piracicaba, POTAFOS, 1986. p.165-274.

MALAVOLTA, E.; VITTI, G.C. \& OLIVEIRA, S.A. Avaliação do estado nutricional das plantas. 2.ed. Piracicaba, POTAFOS, 1997. 310p.

MARSCHNER, H. Mineral nutrition of higher plants. London, Academic Press, 1995. 889p.

MARTINEZ, H.E.P. \& MONNERAT, P.H. Níveis crescentes de alumínio em duas variedades de café cultivadas em solução nutritiva. In: CONGRESSO BRASILEIRO DE CIÊNCIA DO SOLO, 23., Porto Alegre, 1991. Resumo. Porto Alegre, Sociedade Brasileira de Ciência do Solo, 1991. p.109

MARTINEZ, H.E.P.; MENEZES, J.F.S.; SOUZA, R.B.; ALVAREZ V., V.H. \& GUIMARÃES, P.T.G. Faixas críticas de concentrações de nutrientes e avaliação do estado nutricional de cafeeiros em quatro regiões de Minas Gerais. Pesq. Agropec. Bras., 38:703-713, 2003.

MATTIELLO, E.M.; SILVA, I.R.; MAURI, J.; MEIRELES, P.G.; MATIELLO, J.B.; PEREIRA, M.G. \& ZONTA, E. Crescimento radicular e absorção de cálcio, fósforo e alumínio em cafeeiros cultivados em solução nutritiva sob efeito do alumínio.In: FERTBIO-2004, Lages-SC, 2004. Resumo expandido. Lages, Sociedade Brasileira de Ciência do Solo, 2004. CD-ROM

MAURI, J.; MATIELO, J.B.; MEIRELES, P.G.; MATIELLO, E.M.; ZONTA, E. \& PEREIRA, M.G. Efeito do alumínio no desenvolvimento de Coffea canephora e Coffea arábica cultivados em solução nutritiva. In: FERTBIO-2004, LagesSC, 2004. Resumo expandido. Lages, Sociedade Brasileira de Ciência do Solo, 2004. CD-ROM

PAVAN, M.A. \& BINGHAM, F.T. Níveis de alumínio em cafeeiros cultivados em solução nutritiva. Pesq. Agropec. Bras.,17:9:1293-1302, 1982

PAVAN, M.A.; BINGHAM, F.T. \& PRATT, P.F. Toxicity of aluminum to coffee in ultisols and oxisols ameded with $\mathrm{CaCO}_{3}, \mathrm{MgCO}_{3}$ and $\mathrm{CaSO}_{4} .2 \mathrm{H}_{2} \mathrm{O}$. J. Sci. Soc. Am., 46:12011207, 1982.

RAIJ, B. van. Fertilidade do solo e adubação. Campinas, Agronômica Ceres, POTAFOS, 1991. 343p. 
RAJU, P.S.; CLARCK, R.B.; MAITI, R.K. \& MARANVILLE, J.W. Phosphorus uptake, distribution and use by glossy and nonglossy sorghum. J. Plant Nutr., 10:2017-2024, 1987.

RENGEL, Z.; PINEROS, M. \& TESTER, M. Transmembrane calcium fluxes during Al stress. Plant Soil, 171:125-130, 1995.

RODRIGUES, L.A.; MARTINEZ, H.E.P.; NEVES, J.C.L.; NOVAIS, R.F. \& MENDONÇA, S.M. Growth response of coffee tree shoots and roots to subsurface liming. Plant Soil, 234:207-214, 2001.

SIDDIQUI, M.Y \& GLASS, A.D.M. Utilization index: a modified approach to the estimation and comparation of nutrient utilization efficiency in plants. J. Plant. Nutr., 4:289-302, 1981.
SILVA, I.R.; SMYTH, T.J.; BARROS, N.F. \& NOVAIS, R.F. Physiological aspects of aluminum toxicity and tolerance in plants. In: ALVAREZ V., V.H.; SCHAEFER, C.E.G.R.; BARROS, N.F.; MELLO, J.W.V. \& COSTA, L.M., eds. Tópicos de ciência do solo. Viçosa, MG, Sociedade Brasileira de Ciência do Solo, 2002. v 2. p 277-336.

TAYLOR, G.J. The physiology of aluminum tolerance in higher plants. Comm. Soil Sci. Plant Anal., 19:1179-1194, 1988.

VETTORI, L. Métodos de análise de solo. Rio de Janeiro, Ministério da Agricultura, Equipe de Pedologia e Fertilidade do Solo, 1969. 24p. (Boletim Técnico)

WAGATSUNAGA, T. Characteristics of upward translocation of aluminum in plants. Soil Sci. Plant Nutr., 30:345-358, 1984. 